Review

\title{
Skeletal Muscle Mitochondrial Energetic Efficiency and Aging
}

\author{
Raffaella Crescenzo, Francesca Bianco, Arianna Mazzoli, Antonia Giacco, \\ Giovanna Liverini and Susanna Iossa *
}

Department of Biology, University of Naples "Federico II”, Napoli I-80126, Italy; E-Mails: rcrescen@unina.it (R.C.); francibianco@yahoo.it (F.B.); arimazzoli@hotmail.it (A.M.); antonia.giacco@hotmail.it (A.G.); liverini@unina.it (G.L.)

* Author to whom correspondence should be addressed; E-Mail: susiossa@unina.it; Tel.: +39-081-253-8111; Fax: +39-081-679-233.

Academic Editor: Lars Olson

Received: 9 February 2015 / Accepted: 5 May 2015 / Published: 11 May 2015

\begin{abstract}
Aging is associated with a progressive loss of maximal cell functionality, and mitochondria are considered a key factor in aging process, since they determine the ATP availability in the cells. Mitochondrial performance during aging in skeletal muscle is reported to be either decreased or unchanged. This heterogeneity of results could partly be due to the method used to assess mitochondrial performance. In addition, in skeletal muscle the mitochondrial population is heterogeneous, composed of subsarcolemmal and intermyofibrillar mitochondria. Therefore, the purpose of the present review is to summarize the results obtained on the functionality of the above mitochondrial populations during aging, taking into account that the mitochondrial performance depends on organelle number, organelle activity, and energetic efficiency of the mitochondrial machinery in synthesizing ATP from the oxidation of fuels.
\end{abstract}

Keywords: mitochondria; aging; skeletal muscle; proton leak

\section{Introduction}

Increasing age leads to a decline in cell functionality, generally termed as "aging" [1]. All tissue and organs of the body are involved in the phenomenon of aging, but the extent of the cellular impairment is greatly variable, since post-mitotic tissues are the most sensitive targets of the aging process [2]. Among 
the latter, skeletal muscle tissue is profoundly affected during aging, and its functional decline is characterized by a progressive atrophy, that becomes more severe with advancing age and that from a certain point onwards can lead to mobility impairment, increased risk of falls, and physical frailty $[3,4]$. The loss of function in skeletal muscle is also responsible for the development of age-associated insulin resistance and related metabolic disturbances [5-9]. For these reasons, understanding the mechanisms underlying aging in skeletal muscle is fundamental for promotion of health and mobility in the elderly. To this end, the most used animal model of human aging is represented by ad libitum fed caged rodents, that exhibit a sedentary lifestyle and unrestricted diet, and that develop an age-induced spontaneous obesity and insulin resistance already at 4 months of age [7].

Although the loss of skeletal muscle protein mass during aging could at least partially explain the decline in muscle performance, a role for mitochondria has been postulated in the aging process [10,11]. In fact, mitochondria have a major role in energetic homeostasis by determining ATP availability in the cells. A decrease in mitochondrial function could therefore cause an inability to meet cellular ATP demands, so that skeletal muscle cells lose their capacity to adapt to physiological stress imposed across the entire lifespan [12]. In addition, dysfunctional mitochondria could contribute to the development of age-induced insulin resistance [13], since mitochondrial oxidative capacity has been considered a good predictor of insulin sensitivity [14].

One important issue that should be taken into account when studying mitochondria in skeletal muscle is that the mitochondrial population is heterogeneous, composed of mitochondria located either beneath the sarcolemmal membrane (subsarcolemmal, SS) or between the myofibrils (intermyofibrillar, IMF) [15] (Figure 1). Since these two mitochondrial populations exhibit different energetic characteristics and can be differently affected by physiological stimuli [16], it is important that both are separately studied. Therefore, the purpose of the present review is to summarize the results obtained on the functionality of the above mitochondrial populations during aging, taking into account that the mitochondrial performance depends on organelle number, organelle activity, and energetic efficiency of the mitochondrial machinery in synthesizing ATP from the oxidation of fuels. A search in PubMed of relevant articles was conducted, by using query "skeletal muscle mitochondria and aging", "subsarcolemmal mitochondria and aging", and "intermyofibrillar mitochondria and aging", with the inclusion of related articles by the same groups.

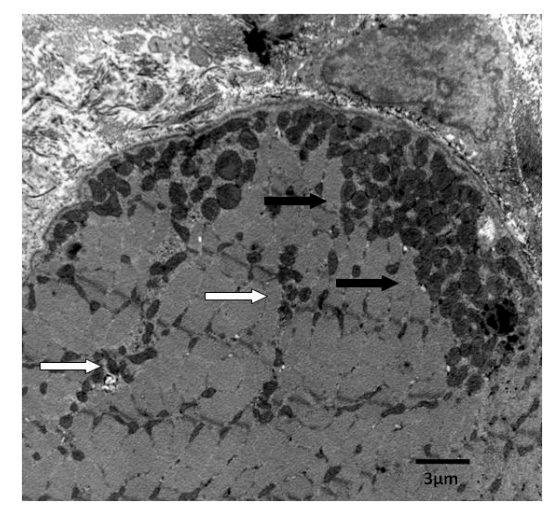

Figure 1. Heterogeneous mitochondrial populations in skeletal muscle cells. Scale bar $=3 \mu \mathrm{m}$. White arrows $=$ Intermyofibrillar mitochondria; Black arrows $=$ Subsarcolemmal mitochondria . 


\section{Age-Related Changes in Mitochondria}

Although many studies have addressed the issue of mitochondrial performance attenuation during aging in skeletal muscle, the obtained results are contradictory, with some papers reporting impairment of mitochondria with increasing age [17-23] and others showing no age-induced change [24,25], underscoring the complexity of understanding in this area.

The discrepancy between the published data can partly be explained by differences in experimental approach. However, even when comparing the results obtained with similar methodological approach, divergent outcomes are evident. For example, among the studies that have measured the activity of enzymatic complexes, such as citrate synthase and electron transport chain complexes, to obtain indirect insights into the energy producing (respiratory) capacity of the mitochondria, some of them have reported an age-dependent decrease in aging muscle [26-28] while other studies found no variation [29] or reported a variable response in different muscles [30]. An alternative approach used to study mitochondrial alteration in aging muscle is assessing total mitochondrial content. Again, some studies reported that mitochondrial content is reduced in aging muscle [31,32] and others found no change [21,33-35]. There is however consensus on the finding that aging skeletal muscle has a blunted capacity for generation of new mitochondria in response to both endurance exercise training [36] and chronic electrical stimulation [37,38].

To our knowledge, studies carried out specifically on mitochondria located beneath the sarcolemmal membrane (SS) and between the myofibrils (IMF) from skeletal muscle during aging are scarce. In a pioneering research, Farrar et al. [39] found that ADP-stimulated respiration did not change with increasing age in IMF and SS mitochondria, while aging decreased the amount of IMF proteins. Chabi et al. [32] studied senescent (3 years) rats and have found that in SS and IMF mitochondria the capacity for ATP production was reduced, as a result of diminished mitochondrial content per gram of muscle. Drew et al. [40] found a decrease in ATP production between 12 and 26 months of age in SS mitochondria from gastrocnemius muscle in Fisher rats. Very few studies specifically addressed the differential regulation of SS and IMF mitochondria with aging in humans. These studies evaluated the SS and IMF mitochondrial content of skeletal muscle in young and old men and reported no age-dependent change in both mitochondrial populations [34,41]. From all the above results, it emerges a differential effect of aging on SS and IMF mitochondria, at least in animal models. More studies on humans are needed to validate the differential effect of aging on the two mitochondrial populations. In addition, studies on changes in mitochondrial function induced by aging or other physiological stimuli should be carried out on the two different mitochondrial populations existing in skeletal muscle.

\section{Mitochondrial Energetic Efficiency during Aging}

From the analysis of the published results on the differential effect of aging on IMF and SS mitochondria, no clear picture can be obtained, partly because of differences in the experimental approach used to evaluate mitochondrial function. The mitochondrial performance depends on organelle number, organelle activity, and energetic efficiency of the mitochondrial machinery in synthesizing ATP from the oxidation of fuels. In addition, it is well known that the amount of fuels oxidized by the cell is dictated mainly by ATP turnover rather than by mitochondrial oxidative capacity and therefore a 
decrease in mitochondrial capacity and/or number becomes more important when cells increase their metabolic activity, i.e., during contraction, while an increased mitochondrial efficiency still alter the amount of oxidized fuels also at rest (Figure 2). The efficiency with which dietary calories are converted to ATP is determined by the degree of coupling of oxidative phosphorylation. If the respiratory chain is highly efficient at pumping protons out of the mitochondrial inner membrane, and the ATP synthesis is highly efficient at converting the proton flow through its proton channel into ATP (from ADP), then the mitochondria will generate maximum ATP and minimum heat per calorie. In contrast, if the efficiency of proton pumping is reduced and/or more protons are required to make each ATP molecule, then each calorie will yield less ATP.

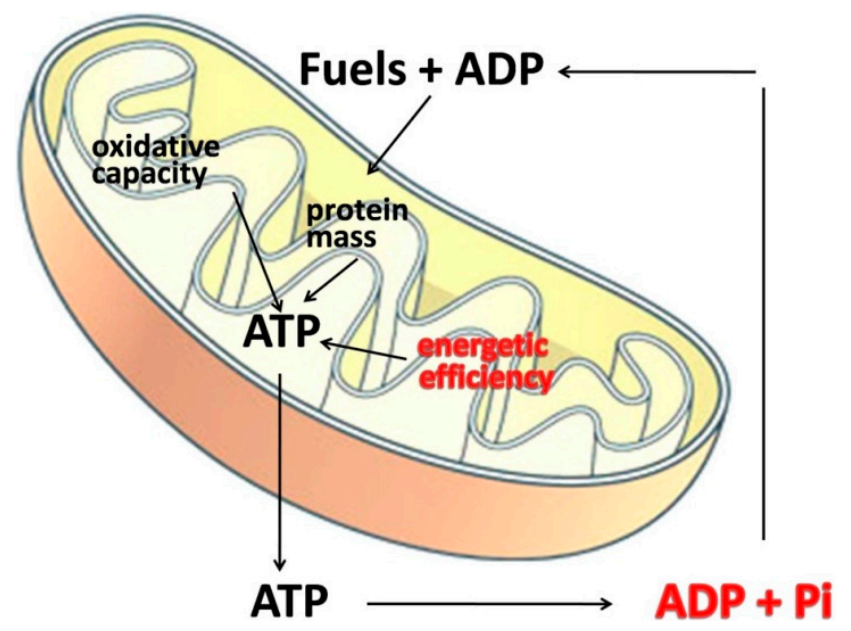

Figure 2. Factors affecting cellular fuel oxidation. The amount of burned fuels mainly depends on mitochondrial energetic efficiency and ATP turnover (in red). Mitochondrial mass and oxidative capacity are less important because mitochondria are thought to have a much greater capacity to generate ATP than what is usually required [42].

The main point of regulation of the oxidative phosphorylation efficiency [43] is represented by the degree of coupling between oxygen consumption and ATP synthesis, which is always lower than 1 and can vary according to the metabolic needs of the cell [44]. Among the factors, which affect mitochondrial degree of coupling, an important role is played by the permeability of the mitochondrial inner membrane to $\mathrm{H}^{+}$ions (leak). It is now well known that mitochondrial inner membrane exhibit a basal proton leak pathway, whose contribution to the basal metabolic rate in rats has been estimated to be about $20 \%-25 \%$ [45]. In addition, it is well known that fatty acids can act as natural uncouplers of oxidative phosphorylation, by generating a fatty acid-dependent proton leak pathway [46-48], which is a function of the amount of unbound fatty acids in the cell.

The issue of mitochondrial efficiency has been explored in humans in vivo, and it has been found that the effect of aging is fiber type-dependent. In fact, in mildly uncoupled fiber types (i.e., tibialis anterior) no age effect is evident, while a substantial uncoupling takes place with aging in well coupled fiber types (i.e., dorsal interosseous) [49]. Due to the in vivo conditions, these studies do not distinguish between SS and IMF mitochondria. Since aging has been show to selectively affect IMF but not SS mitochondria in heart [50], and taking into account the above considerations, a study was carried out on the putative changes in mitochondrial performance and efficiency during aging in SS and IMF mitochondria. In the 
transition from young adulthood (60 days) to adulthood (180 days), SS and IMF skeletal muscle mitochondria displayed an increase in the degree of coupling and efficiency, as well as a decreased fatty acid dependent proton leak [51]. The above modifications in mitochondrial performance occurred concomitantly with an increase in whole body lipids and plasma non-esterified fatty acids (NEFA) [51], suggesting a link between skeletal muscle increased mitochondrial efficiency and metabolic impairments.

These results were extended to the evaluation of how the progression of aging affects skeletal muscle mitochondrial function by measuring mitochondrial respiratory capacity and proton leak in SS and IMF mitochondria from adult (six months) and old (two years) rats [52]. A significant decrease in oxidative capacity was found in skeletal muscle homogenates, as well as in SS and IMF mitochondria from old rats. Oxidative capacity measured in the homogenate reflects the product of mitochondrial mass and activity, while oxidative capacity in isolated organelles depends only on mitochondrial activity, and therefore the similar age-induced decrease in oxidative capacity in homogenates and isolated mitochondria found in old rats could be indicative of a lack of changes in mitochondrial mass. A decreased mitochondrial mass has been found in senescent rats (36 months old) [32], thus suggesting that an impairment of mitochondrial biogenesis occurs in late aging and/or it takes place selectively in specific muscles, such as gastrocnemius, whose mitochondrial mass has been found decreased in old rats $[20,53]$.

Both mitochondrial populations from old rats exhibited a significant decrease in proton leak [52], suggesting that with increasing age the efficiency of oxidative phosphorylation increases both in SS and IMF mitochondria. Similar results have been obtained in vivo in aged rat skeletal muscle, where a trend for a higher coupling efficiency was found [20]. Skeletal muscle cells in sedentary laboratory rats operate in conditions of low ADP availability, near to state 4 (when no ADP is available), with a high contribution of proton leak to total oxygen consumed by mitochondria [54], and therefore the decreased proton leak found in SS and IMF mitochondria from old rats is physiologically relevant. When mitochondria are more efficient, less substrates are oxidized to obtain ATP. Therefore, the increased mitochondrial coupling in skeletal muscle could contribute to the decreased energy expenditure that is evident even after the decrease in lean mass has been taken into account and that is at the basis of age-induced obesity, since skeletal muscle energy metabolism accounts for about 30\% of whole body energy expenditure in resting conditions [45].

One interesting question is: What could be the impact of the increased mitochondrial coupling on ATP yield? Although it is very difficult to calculate the theoretical difference in ATP yield per calorie in highly efficient $v s$. inefficient proton pumping, a rough estimate could be obtained using a published estimate of the control value of proton leak on $P / O$ ratio in skeletal muscle [55]. This value is reported to be -0.72 [55], so we can calculate that the $40 \%$ decrease in proton leak found in old rats [52] would result in a $29 \%$ increase in $P / O$ ratio, and therefore in the amount of ATP obtained per unit of oxygen consumed. However, when mitochondria are more coupled, ATP is produced at a slower rate $[44,56]$, that could be unable to meet cellular energy demands, especially during skeletal muscle contraction. In fact, in elderly subjects it has been found that a lower speed of ATP production is associated with higher fatigability [57].

Another deleterious consequence of reduced substrate burning in skeletal muscle could be intracellular triglyceride accumulation and lipotoxicity, since NEFA serum levels are significantly higher in older rats. In fact, in conditions of increased plasma NEFA, more fatty acids enter in the cells and if they are 
poorly oxidized, they accumulate intracellularly in myocytes mainly as long-chain fatty acyl-CoA, monoacylglcyerol, diacylglycerol, phosphatidic acid, triacylglycerol and ceramide. Among these fatty acid derivatives, high intramyocellular levels of diacylglycerol and ceramides are directly associated with insulin resistance [58]. The metabolic implications of increased mitochondrial energetic efficiency are summarized in Figure 3.

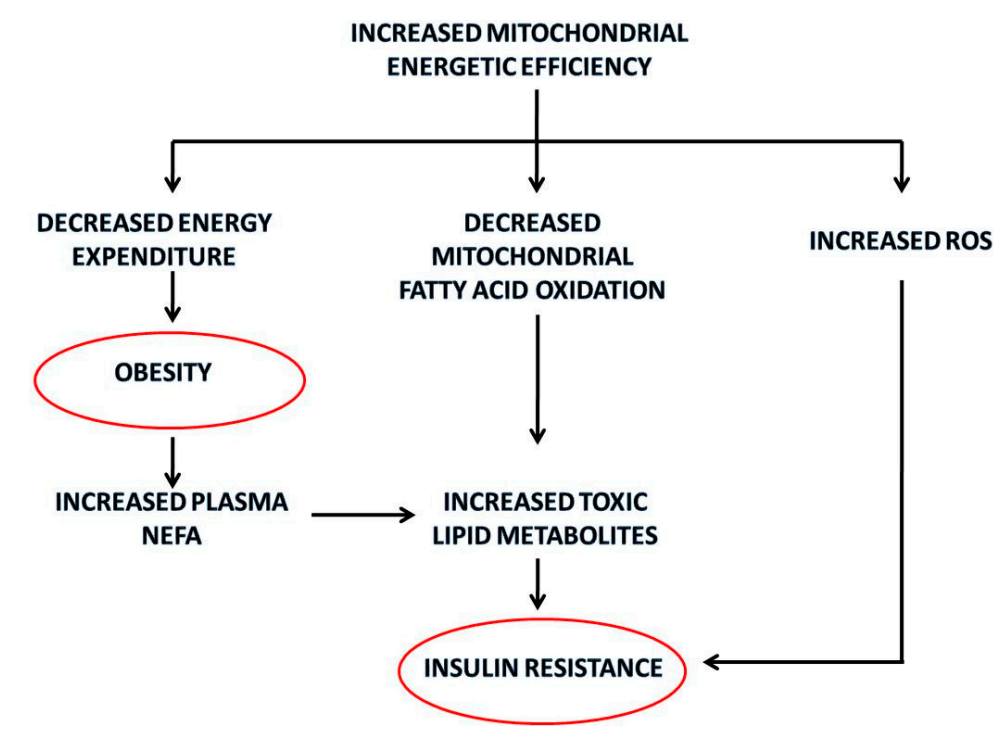

Figure 3. Summary of the metabolic implications of the increased mitochondrial energetic efficiency in skeletal muscle with aging. The red circles identify the pathological outcomes.

\section{Oxidative Stress in Aging Mitochondria}

Reactive oxygen species (ROS) production increases when mitochondrial potential is higher [59,60], and therefore increased mitochondrial energetic efficiency could induce a condition of increased oxidative stress. In fact, one of the postulated physiological roles for the uncoupling effect of fatty acids is to maintain mitochondrial membrane potential below the critical threshold for ROS production, especially in situations of low rates of ATP turnover, such as in resting skeletal muscle [61]. It has been proposed that ROS generation during the normal oxidative activity of mitochondria leads to damage of lipids, proteins and DNA, especially in postmitotic tissues, such as skeletal muscle, and that this oxidative damage is at the basis of the biological phenomenon of cellular aging [10,11]. Therefore, the increased coupling of mitochondrial oxidative phosphorylation found in 180 day-old rats [51] could led to the oxidative damage of skeletal muscle cell, detectable later in life. However, the increased uncoupling protein 3 (UCP3) protein content found in SS and IMF mitochondria from 180 days old rats could be involved in the protection from oxidative damage [62,63]. In fact, it has been proposed that UCP3 translocates fatty acid peroxides from the inner to the outer membrane leaflet, thus preserving macromolecules from being oxidized by very aggressive fatty acid peroxides [62,63], while its uncoupling effect is considered very low, due to its low content in skeletal muscle mitochondria [64]. Therefore, the up-regulation of UCP3 during aging could buffer oxidative damage, that otherwise could be even higher.

In 2 year-old rats, an increase in the degree of lipid peroxidation was found only in SS mitochondria, although the decrease in proton leak is the same in both mitochondrial populations [52], while 
Chabi et al. [32] studied senescent (3 years) rats and found that ROS production was enhanced in SS and IMF mitochondria. The differential susceptibility to oxidative stress of SS and IMF mitochondria could depend on their different content of UCP3. In fact, a significant increase in UCP3 content was evident in SS and IMF mitochondria from old rats, but it was more marked in IMF mitochondria (about 10-fold) than in SS mitochondria (about 5-fold). Therefore, the lower increase in UCP3 content in SS mitochondria is probably the cause of the higher oxidative damage found in this mitochondrial population, while IMF mitochondria seem more protected by oxidative damage by the marked up-regulation of UCP3, so preserving the capacity to produce ATP for muscle contraction. One could speculate that, as aging proceeds, SS mitochondria undergo progressive oxidative damage with loss of functional activity. In fact, in senescent (30-36 month-old) animals, an increase in mitochondrial proton leak [65], a decrease in mitochondrial coupling [66] or a decrease in mitochondrial membrane potential in SS but not in IMF mitochondria has been found [32]. In conclusion, the increased coupling of SS mitochondria causes an increase of the oxidative damage in this mitochondrial population, that is located beneath the plasma membrane and provides ATP for membrane transports and signal transduction pathways [67]. On the other hand, IMF mitochondria, that provide ATP for muscle contraction, seems to be more protected from oxidative damage, and could thus increase their oxidative capacity and density in response to endurance training even in old age [68].

A decreased UCP3 content has been found in skeletal muscle mitochondria from old rats $[31,69,70]$, but these results have been obtained using Fischer 344 rats, a rat strain that gain weight only moderately with age compared with other strains (i.e., Sprague-Dawley, Wistar, Long Evans) [8]. Therefore, it can be hypothesized that the regulation of UCP3 with aging in skeletal muscle mitochondria is strain-dependent. Therefore, it is possible that age-induced oxidative damage in skeletal muscle and age-induced obesity are intimately linked. Further studies on the degree of obesity and oxidative damage induced by aging in different strains and species are needed to substantiate the hypothesis.

\section{Conclusions}

In the rat model of human obesity the progression of aging is accompanied by an increased efficiency of SS and IMF mitochondria but an increased oxidative damage occurs only in the SS population. Therefore, a differential susceptibility of SS and IMF mitochondria to aging-induced damage emerges, although more studies on humans are needed to validate the differential effect of aging on the two mitochondrial populations. These observations also indicate that studies on changes in mitochondrial function induced by aging or other physiological stimuli should be carried out on the two different mitochondrial populations existing in skeletal muscle.

\section{Acknowledgments}

The work was supported by University "Federico II" of Naples.

\section{Author Contributions}

All authors contributed to the writing of this review. 


\section{Conflicts of Interest}

The authors declare no conflict of interest.

\section{References}

1. Figueiredo, P.A.; Mota, M.P.; Appell, H.J.; Duarte, J.A. The role of mitochondria in aging of skeletal muscle. Biogerontology 2008, 9, 67-84.

2. Kwong, L.K.; Sohal, R.S. Age-related changes in activities of mitochondrial electron transport complexes in various tissues of the mouse. Arch. Biochem. Biophys. 2000, 373, 16-22.

3. Cruz-Jentoft, J.; Landi, F.; Topinkova, E.; Michel, J.P. Understanding sarcopenia as a geriatric syndrome. Curr. Opin. Clin. Nutr. Metab. Care 2010, 13, 1-7.

4. Marzetti, E.; Calvani, R.; Cesari, M.; Buford, T.W.; Lorenzi, M.; Behnke, B.J.; Leeuwenburgh, C. Mitochondrial dysfunction and sarcopenia of aging: From signaling pathways to clinical trials. Int. J. Biochem. Cell Biol. 2013, 45, 2288-2301.

5. Shimokata, H.; Tobin, J.; Muller, D.C.; Elahi, D.; Coon, P.J.; Andres, R. Studies in the distribution of body fat: 1. Effects of age, sex and obesity. J. Gerontol. 1989, 44, M66-M73.

6. Reaven, G.M.; Reaven, E.P. Age, glucose intolerance, and non-insulin dependent diabetes mellitus. J. Am. Geriatr. Soc. 1985, 33, 286-290.

7. Barzilai, N.; Rossetti, L. Relationship between changes in body composition and insulin responsiveness in models of the aging rat. Am. J. Physiol. 1995, 269, E591-E597.

8. Larkin, L.M.; Reynolds, T.H.; Supiano, M.A.; Kahn, B.B.; Halter, J.B. Effect of aging and obesity on insulin responsiveness and glut-4 glucose transporter content in skeletal muscle of Fisher $344 \times$ Brown Norway rats. J. Gerontol. 2001, 56, B486-B492.

9. Iossa, S.; Mollica, M.P.; Lionetti, L.; Crescenzo, R.; Botta, M.; Barletta, A.; Liverini, G. Acetyl-L-carnitine supplementation differently influences nutrient partitioning, serum leptin concentration and skeletal muscle mitochondrial respiration in young and old rats. J. Nutr. 2002, 132, 636-642.

10. Lenaz, G. Role of mitochondria in oxidative stress and ageing. Biochim. Biophyis. Acta 1998, 1366, $53-67$.

11. Lenaz, G.; D’Aurelio, M.; Merlo Pich, M.; Genova, M.L.; Ventura, B.; Bovina, C.; Formiggini, G.; Parenti Castelli, G. Mitochondrial bioenergetics in aging. Biochim. Biophys. Acta 2000, 1459, 397-404.

12. Shigenaga, M.K.; Hagen, T.M.; Ames, B.N. Oxidative damage and mitochondrial decay in aging. Proc. Natl. Acad. Sci. USA 1994, 91, 10771-10778.

13. Petersen, K.F.; Befroy, D.; Dufour, S.; Dziura, J.; Arijan, C.; Rothman, D.L.; DiPietro, L.; Cline, G.W.; Shulman, G.I. Mitochondrial disfunction in the elderly: Possible role in insulin resistance. Science 2003, 300, 1140-1142.

14. Bruce, C.R.; Anderson, M.J.; Carey, A.L.; Newman, D.G.; Bonen, A.; Kriketos, A.D.; Cooney, G.J.; Hawley, J.A. Muscle oxidative capacity is a better predictor of insulin sensitivity than lipid status. J. Clin. Endocrinol. Metab. 2003, 88, 5444-5451.

15. Drew, B.; Leeuwenburgh, C. Ageing and subcellular distribution of mitochondria: Role of mitochondrial DNA deletions and energy production. Acta Physiol. Scand. 2004, 182, 333-341. 
16. Mollica, M.P.; Lionetti, L.; Crescenzo, R.; D’Andrea, E.; Ferraro, M.; Liverini, G.; Iossa, S. Heterogeneous bioenergetic behaviour of subsarcolemmal and intermyofibrillar mitochondria in fed and fasted rats. Cell. Mol. Life Sci. 2006, 63, 358-366.

17. Figueiredo, P.A.; Powers, S.K.; Ferreira, R.M.; Appell, H.J.; Duarte, J.A. Aging impairs skeletal muscle mitochondrial bioenergetic function. J. Gerontol. A Biol. Sci. Med. Sci. 2009, 64, 21-33.

18. Trounce, I.; Byrne, E.; Marzuki, S. Decline in skeletal muscle mitochondrial respiratory chain function: Possible factor in ageing. Lancet 1989, 1, 637-639.

19. Conley, K.E.; Jubrias, S.A.; Esselman, P.C. Oxidative capacity and aging in human muscle. J. Physiol. 2000, 526, 203-210.

20. Gouspillou, G.; Bourdel-Marchasson, I.; Rouland, R.; Calmettes, G.; Biran, M.; Deschodt-Arsac, V.; Miraux, S.; Thiaudiere, E.; Pasdois, P.; Detaille, D.; et al. Mitochondrial energetics is impaired in vivo in aged skeletal muscle. Aging Cell 2014, 13, 39-48.

21. Gouspillou, G.; Sgarioto, N.; Kapchinsky, S.; Purves-Smith, F.; Norris, B.; Pion, C.H.; Barbat-Artigas, S.; Lemieux, F.; Taivassalo, T.; Morais. J.A.; et al. Increased sensitivity to mitochondrial permeability transition and myonuclear translocation of endonuclease $\mathrm{G}$ in atrophied muscle of physically active older humans. FASEB J. 2014, 28, 1621-1633.

22. Gouspillou, G.; Bourdel-Marchasson, I.; Rouland, R.; Calmettes, G.; Franconi, J.M.; Deschodt-Arsac, V.; Diolez, P. Alteration of mitochondrial oxidative phosphorylation in aged skeletal muscle involves modification of adenine nucleotide translocator. Biochim. Biophys. Acta 2010, 1797, 143-151.

23. Picard, M.; Ritchie, D.; Thomas, M.M.; Wright, K.J.; Hepple, R.T. Alterations in intrinsic mitochondrial function with aging are fiber type-specific and do not explain differential atrophy between muscles. Aging Cell 2011, 10, 1047-1055.

24. Rasmussen, U.F.; Krustrup, P.; Kjaer, M.; Rasmussen, H.N. Experimental evidence against the mitochondrial theory of aging. A study on isolated human skeletal muscle mitochondria. Exp. Gerontol. 2003, 38, 877-886.

25. Rasmussen, U.F.; Krustrup, P.; Kjaer, M.; Rasmussen, H.N. Human skeletal muscle mitochondrial metabolism in youth and senescence: No signs of functional changes of ATP formation and mitochondrial capacity. Pflug. Arch. 2003, 446, 270-278.

26. Desai, V.G.; Weindruch, R.; Hart, R.W.; Feuers, R.J. Influences of age and dietary restriction on gastrocnemius electron transport system activities in mice. Arch. Biochem. Biophys. 1996, 333, $145-151$.

27. Hagen, J.L.; Krause, D.J.; Baker, D.J.; Fu, M.; Tarnopolsky, M.A.; Hepple, R.T. Skeletal muscle aging in F344BN F1-hybrid rats: I. Mitochondrial dysfunction contributes to the age-associated reduction in $\mathrm{VO}_{2 \max }$ J. Gerontol. 2004, 59, 1099-1110.

28. Lombardi, A.; Silvestri, E.; Cioffi, F.; Senese, R.; Lanni, A.; Goglia, F.; de Lange, P.; Moreno, M. Defining the trascriptomic and proteomic profiles of rat ageing skeletal muscle by the use of a cDNA array, 2D- and Blue native-PAGE approach. J. Proteomics 2009, 72, 708-721.

29. Barrientos, A.; Casademont, J.; Rotig, A.; Miro, O.; Urbano-Marquez, A.; Rustin, P.; Cardellach, F. Absence of relationship between the level of electron transport chain activities and aging in human skeletal muscle. Biochem. Biophys. Res. Commun. 1996, 229, 536-539. 
30. Lyons, C.N.; Mathieu-Costello, O.; Moyes, C.D. Regulation of skeletal muscle mitochondrial content during aging. J. Gerontol. 2006, 61, 3-13.

31. Kerner, J.; Turkaly, P.J.; Minkler, P.E.; Hoppel, C.L. Aging skeletal muscle mitochondria in the rat: Decreased uncoupling protein-3 content. Am. J. Physiol. 2001, 281, E1054-E1062.

32. Chabi, B.; Ljubicic, V.; Menzies, K.J.; Huang, J.H.; Saleem, A.; Hood, D.A. Mitochondrial function and apoptotic susceptibility in aging skeletal muscle. Aging Cell 2008, 7, 2-12.

33. Mathieu-Costello, O.; Ju, Y.; Trejo-Morales, M.; Cui, L. Greater capillary-fiber interface per fiber mitochondrial volume in skeletal muscles of old rats. J. Appl. Physiol. 2005, 99, 281-289.

34. Callahan, D.M.; Bedrin, N.G.; Subramanian, M.; Berking, J.; Ades, P.A.; Toth, M.J.; Miller, M.S. Age-related structural alterations in human skeletal muscle fibers and mitochondria are sex specific: Relationship to single-fiber function. J. Appl. Physiol. 2014, 116, 1582-1592.

35. Konopka, R.; Suer, M.K.; Wolff, C.A.; Harber, M.P. Markers of human skeletal muscle mitochondrial biogenesis and quality control: Effects of age and aerobic exercise training. J. Gerontol. 2014, 69, 371-378.

36. Betik, C.; Thomas, M.M.; Wright, K.J.; Riel, C.D.; Hepple, R.T. Exercise training from late middle age until senescence does not attenuate the declines in skeletal muscle aerobic function. Am. J. Physiol. 2009, 297, R744-R755.

37. Ljubicic, V.; Hood, D.A. Diminished contraction-induced intracellular signaling towards mitochondrial biogenesis in aged skeletal muscle. Aging Cell 2009, 8, 394-404.

38. Ljubicic, V.; Joseph, A.M.; Adhihetty, P.J.; Huang, J.H.; Saleem, A.; Uguccioni, G.; Hood, D.A. Molecular basis for an attenuated mitochondrial adaptive plasticity in aged skeletal muscle. Aging 2009, 1, 818-830.

39. Farrar, R.P.; Martin, T.P.; Ardies, C.M. The interaction of aging and endurance exercise upon the mitochondrial function of skeletal muscle. J. Gerontol. 1981, 36, 642-647.

40. Drew, B.; Phaneuf, S.; Dirks, A.; Selman, C.; Gredilla, R.; Lezza, A.; Barja, G.; Leeuwenburgh, C. Effects of aging and caloric restriction on mitochondrial energy production in gastrocnemius muscle and heart. Am. J. Physiol. 2003, 284, R474-R480.

41. Nielsen, J.; Suetaa, C.; Hvid, L.G.; Schroder, H.D.; Aagaard, P.; Orteblad, N. Subcellular localization-dependent decrements in skeletal muscle glycogen and mitochondria content following short-term disuse in young and old men. Am. J. Physiol. 2010, 299, E1053-E1060.

42. Holloszy, J.O. Skeletal muscle "mitochondrial deficiency" does not mediate insulin resistance. Am. J. Clin. Nutr. 2009, 89, 463S-466S.

43. Kadenbach, B. Intrinsic and extrinsic uncoupling of oxidative phosphorylation. Biochim. Biophys. Acta 2003, 1604, 77-94.

44. Stucki, J.W. The optimal efficiency and the economic degrees of coupling of oxidative phosphorylation. Eur. J. Biochem. 1980, 109, 269-283.

45. Rolfe, D.F.S.; Brown, G.C. Cellular energy utilisation and molecular origin of standard metabolic rate in mammals. Physiol. Rev. 1997, 77, 731-758.

46. Skulachev, V.P. Fatty acid circuit as a physiological mechanism of uncoupling of oxidative phosphorylation. FEBS Lett. 1991, 294, 158-162. 
47. Jezek, P.; Engstova, H.; Zackova, M.; Vercesi, A.E.; Costa, A.D.T.; Arruda, P.; Garlid, K.D. Fatty acid cycling mechanism and mitochondrial uncoupling proteins. Biochim. Biophys. Acta 1998, $1365,319-327$.

48. Soboll, S.; Grundel, S.; Schwabe, U.; Scholtz, R. Influence of fatty acids on energy metabolism. 2. Kinetics of changes in metabolic rates and changes in subcellular adenine nucleotide contents and $\mathrm{pH}$ gradients following addition of octanoate and oleate in perfused rat liver. Eur. J. Biochem. 1984, $141,231-236$.

49. Amara, C.E.; Shankland, E.G.; Jubrias, S.A.; Marcinek, D.J.; Kushmerick, M.J.; Conley, K.E. Mild mitochondrial uncoupling impacts cellular aging in human muscles in vivo. Proc. Natl. Acad. Sci. USA 2007, 104, 1057-1062.

50. Fannin, S.W.; Lesnefsky, E.J.; Slabe, T.J.; Hassan, M.O.; Hoppel, C.L. Aging selectively decreases oxidative capacity in rat heart interfibrillar mitochondria. Arch. Biochem. Biophys. 1999, 372, 399-407.

51. Iossa, S.; Mollica, M.P.; Lionetti, L.; Crescenzo, R.; Tasso, R.; Liverini, G. A possible link between skeletal muscle mitochondrial efficiency and age-induced insulin resistance. Diabetes 2004, 53, 2861-2866.

52. Crescenzo, R.; Bianco, F.; Mazzoli, A.; Giacco, A.; Liverini, G.; Iossa, S. Alterations in proton leak, oxidative status and uncoupling protein 3 content in skeletal muscle subsarcolemmal and intermyofibrillar mitochondria in old rats. BMC Geriatr. 2014, 14, 79.

53. Koltai, E.; Hart, N.; Taylor, A.W.; Goto, S.; Ngo, J.K.; Davies, K.J.A.; Radak, Z. Age-associated declines in mitochondrial biogenesis and protein quality control factors are minimized by exercise training. Am. J. Physiol. 2012, 303, R127-R134.

54. Hafner, R.P.; Brown, G.C.; Brand, M.D. Analysis of the control of respiration rate, phosphorylation rate, proton leak rate and protonmotive force in isolated mitochondria using the "top-down" approach of metabolic control theory. Eur. J. Biochem. 1990, 188, 313-319.

55. Brand, M.D.; Chien, L.F.; Ainscow, E.K.; Rolfe, D.F.S.; Porter, R.K. The causes and functions of mitochondrial proton leak. Biochim. Biophys. Acta 1994, 1187, 132-139.

56. Cairns, C.B.; Walther, J.; Harken, A.H.; Banerjee, A. Mitochondrial oxidative phosphorylation thermodynamic efficiencies reflect physiological organ roles. Am. J. Physiol. 1998, 274, R1376-R1383.

57. Santanasto, A.J.; Glynn, N.W.; Jubrias, S.A.; Conley, K.E.; Boudreau, R.M.; Amati, F.; Mackey, D.C.; Simonsick, E.M.; Strotmeyer, E.S.; Coen, P.M.; et al. Skeletal muscle mitochondrial function and fatigability in older adults. J. Gerontol. A Biol. Sci. Med. Sci. 2015, doi:10.1093/gerona/glu134.

58. Stannard, S.R.; Johnson, N.A. Insulin resistance and elevated triglyceride in muscle: More important for survival than thrifty genes? J. Physiol. 2003, 554, 595-607.

59. Korshunov, S.S.; Skulachev, V.P.; Starkov, A.A. High protonic potential actuates a mechanism of production of reactive oxygen species in mitochondria. FEBS Lett. 1997, 416, 15-18.

60. Mailloux, R.J.; Harper, M.E. Uncoupling proteins and the control of mitochondrial reactive oxygen species production. Free Radic. Biol. Med. 2011, 51, 1106-1115.

61. Brand, M.D. Uncoupling to survive? The role of mitochondrial inefficiency in ageing. Exp. Gerontol. 2000, 35, 811-820. 
62. Lombardi, A.; Busiello, R.A.; Napolitano, L.; Cioffi, F.; Moreno, M.; de Lange, P.; Silvestri, E.; Lanni, A.; Goglia, F. UCP3 translocates lipid hydroperoxide and mediates lipid hydroperoxide-dependent mitochondrial uncoupling. J. Biol. Chem. 2010, 285, 16599-16605.

63. Goglia, F.; Skulachev, V.P. A function for novel uncoupling proteins: Antioxidant defence of mitochondrial matrix by translocating fatty acid peroxides from the inner to the outer membrane leaflet. FASEB J. 2003, 17, 1585-1591.

64. Divakaruni, A.S.; Brand, M.D. The regulation and physiology of mitochondrial proton leak. Physiology 2011, 26, 192-205.

65. Lal, S.B.; Ramsey, J.J.; Monemdjou, S.; Weindruch, R.; Harper, M.E. Effects of caloric restriction on skeletal muscle mitochondrial proton leak in aging rats. J. Gerontol. 2001, 56, B116-B122.

66. Marcinek, D.J.; Schenkman, K.A.; Ciesielski, W.A.; Lee, D.; Conley, K.E. Reduced mitochondrial coupling in vivo alters cellular energetic in aged mouse skeletal muscle. J. Physiol. 2005, 569, 467-473.

67. Hood, D. Plasticity in skeletal, cardiac, and smooth muscle: Contractile activity-induced mitochondrial biogenesis in skeletal muscle. J. Appl. Physiol. 2001, 90, 1137-1157.

68. Beyer, R.E.; Starnes, J.W.; Edington, D.W.; Lipton, R.J.; Compton, R.T.; Kwasman, M.A. Exercise-induced reversal of age-related declines of oxidative reactions, mitochondrial yield, and flavins in skeletal muscle of the rat. Mech. Ageing Dev. 1984, 24, 309-323.

69. Bevilacqua, L.; Ramsey, J.J.; Hagopian, K.; Weindruch, R.; Harper, M.E. Long-term caloric restriction increases UCP3 content but decreases proton leak and reactive oxygen species production in rat skeletal muscle mitochondria. Am. J. Physiol. 2005, 289, E429-E438.

70. Barazzoni, R.; Nair, K.S. Changes in uncoupling protein-2 and -3 expression in aging rat skeletal muscle, liver, and heart. Am. J. Physiol. 2001, 280, E413-E419.

(C) 2015 by the authors; licensee MDPI, Basel, Switzerland. This article is an open access article distributed under the terms and conditions of the Creative Commons Attribution license (http://creativecommons.org/licenses/by/4.0/). 